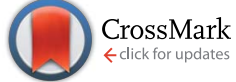

Cite this: RSC Adv., 2016, 6, 45135

\title{
Amphiphilic peptide coated superparamagnetic iron oxide nanoparticles for in vivo MR tumor imaging ${ }^{\dagger}$
}

\author{
Ayse Ozdemir,,$^{a}$ Melis Sardan Ekiz, $\stackrel{+}{a}^{a}$ Alper Dilli, ${ }^{b}$ Mustafa O. Guler ${ }^{\star a}$ \\ and Ayse B. Tekinay ${ }^{\star a}$
}

Magnetic resonance imaging (MRI) is a noninvasive imaging technique that provides high spatial resolution and depth with pronounced soft-tissue contrast for in vivo imaging. A broad variety of strategies have been employed to enhance the diagnostic value of MRI and detect tissue abnormalities at an earlier stage. Superparamagnetic iron oxide nanoparticles (SPIONs) are considered to be suitable candidates for effective imaging due to their small size, versatile functionality and better biocompatibility. Here, we demonstrate that coating SPIONs with proline-rich amphiphilic peptide molecules through noncovalent interactions leads to a water-dispersed hybrid system suitable as an MRI contrast agent. Cellular viability and uptake of amphiphilic peptide coated SPIONs (SPION/K-PA) were evaluated with human vascular endothelial cells (HUVEC) and estrogen receptor (ER) positive human breast adenocarcinoma (MCF-7) cells. The efficiency of SPION/K-PA as MRI contrast agents was analyzed in Sprague-Dawley rats with mammary gland tumors. MR imaging showed that SPION/K-PA effectively accumulated in tumor tissues, enhancing their imaging potential. Although nanoparticles were observed in reticuloendothelial system organs (RES) and especially in the liver and kidney immediately after administration, the MR signal intensity in these organs diminished after $1 \mathrm{~h}$ and nanoparticles were subsequently cleared from these organs within two weeks. Histological observations also validated the accumulation of nanoparticles in tumor tissue at $4 \mathrm{~h}$ and their bioelimination from the organs of both healthy and tumor-bearing rats after

Received 21st March 2016 Accepted 29th April 2016

DOI: $10.1039 / c 6 r a 07380 h$

www.rsc.org/advances two weeks.

\section{Introduction}

Several techniques are currently used for noninvasive medical imaging in medicine to improve the quality of diagnoses and use more precise treatments. ${ }^{1,2}$ Magnetic resonance imaging (MRI) is a non-ionizing, non-invasive imaging technique that provides high spatial resolution and depth with pronounced soft-tissue contrast for in vivo imaging. The differential contrast observed between soft tissues can be adjusted by altering the data acquisition parameters. ${ }^{3}$ Water molecules are the primary source of protons for the generation of MRI signals, although lipids can also function in this capacity. ${ }^{4}$ Since healthy and unhealthy tissues may have different intrinsic relaxation times depending on their physiological environment, specific contrast

\footnotetext{
Institute of Materials Science and Nanotechnology, National Nanotechnology Research Center (UNAM), Bilkent University, 06800, Bilkent, Ankara, Turkey. E-mail: moguler@unam.bilkent.edu.tr; atekinay@bilkent.edu.tr

${ }^{b}$ Diskapi Yildirim Beyazit Training and Research Hospital, 06110, Altindag, Ankara, Turkey

$\dagger$ Electronic supplementary information (ESI) available. See DOI: 10.1039/c6ra07380h

$\ddagger$ These authors contributed equally to this manuscript.
}

can be observed between the site of interest and the surrounding regions at advanced stages of disease. ${ }^{5} \mathrm{~A}$ variety of contrast agents (CAs) have been designed to enhance the diagnostic value of MRI and detect tissue abnormalities at an earlier stage, typically by shortening $T_{1}$ or $T_{2}$ relaxation times and thereby increasing $r_{1}\left(1 / T_{1}\right)$ or $r_{2}\left(1 / T_{2}\right)$ relaxivities. ${ }^{6}$ These contrast agents are promising candidates for highly effective molecular imaging due to their small size and versatile functionality. While positive CAs, which are mainly gadolinium- and manganese-containing agents, lower the $T_{1}$ relaxation time and create a brighter contrast, negative CAs, which are primarily iron-based compounds, shorten the $T_{2}$ relaxation time and result in darker contrast. Because of the detrimental effects associated with the dissociation and accumulation of gadolinium and manganese ions in the body, ${ }^{7}$ considerable research has been performed on the preparation of nontoxic formulations of these metals, as well as on the preparation of non-toxic alternatives to traditional $T_{1}$ contrast agents. Iron oxide-based CAs are one such example of low-toxicity $T_{2}$ contrast agents, as iron is naturally found in human blood and excess iron can be stored as ferritin in the body. Magnetite $\left(\mathrm{Fe}_{3} \mathrm{O}_{4}\right)$ nanoparticles are especially suitable as next-generation imaging agents due to their biocompatibility, biodegradability and low 
level of toxicity. ${ }^{\mathbf{8} 9}$ These iron oxides can also display superparamagnetic properties depending on the crystal structure, size and shape of the synthesized particle, further enhancing their versatility in biomedical applications. ${ }^{\mathbf{1 0}}$

Recent advances in the development of surface modified nanoparticles have increased usage of MRI for cancer diagnosis. ${ }^{11}$ Several groups have reported that tumor specific MRI $T_{2}$ imaging agents are biocompatible, biodegradable and have long blood circulation time, which provide high accumulation in the tumor tissue. ${ }^{12,13}$ As contrast agents, iron oxide nanoparticles provide several opportunities in the accurate diagnosis of cancer. ${ }^{14}$ For example, iron oxide nanocubes encapsulated with polyethylene glycol-phospholipid have been shown to increase sensitivity in tumor imaging compared to Feridex. ${ }^{15}$ Lee and coworkers have developed branched PEI and PEG coated SPIONs that are able to bind to negatively charged plasmid DNA due to the positive charge on their surface and are detectable at the tumor site by MRI. ${ }^{\mathbf{1 6}}$

The MRI contrast agents are administrated intravenously and their solubility and dispersity in water and serum are also essential for their applicability in biomedical applications. ${ }^{\mathbf{1 7}}$ Therefore, a diverse array of surface modification strategies were developed to increase the colloidal stability of CAs, enhance their pharmacokinetic performances and allow facile coupling of biological ligands to their surfaces. ${ }^{18}$ Up to now, ligands possessing anchor groups such as carboxylic acids, phosphines, amines, and catechol were generally utilized to modify the surface of iron oxide nanoparticles through ligand exchange reactions, rendering them water-dispersible. ${ }^{19}$ Depending on the availability of terminal functional groups in the ligand, bioactivity can also be imparted by decorating a chemically active organic coating with different moieties such as cell penetrating or RGD peptides, ${ }^{20,21}$ antibodies,${ }^{22}$ folic acid ${ }^{23}$ and carbohydrates. ${ }^{\mathbf{2 4 , 2 5}}$ Alternatively, amphiphilic ligands such as PEGylated phospholipids, and PEGylated or Pluronic polymers were exploited to form organic shells around the nanoparticle core by physical encapsulation or assembly through van der Waals interactions and/or electrostatic interactions. ${ }^{\mathbf{1 9}}$ Also, coating of hydrophobic iron oxide nanoparticles with hydrophilic amphiphilic peptides were accomplished by noncovalent interactions. ${ }^{26}$ There is a need for iron oxide-based CAs for in vivo applications.

Here, we demonstrate noncovalent coating of SPIONs with proline-rich amphiphilic peptides (SPION/K-PA), and investigated the efficiency of this superparamagnetic, waterdispersible hybrid system in enhancing the MR imaging of tumor tissue in rats. The physicochemical characteristics of SPION/K-PA was investigated through material characterization techniques, while its potential as an MRI contrast agent was assessed in both in vitro and in vivo experiments. In particular, the cellular viability and uptake behavior of the SPION/K-PA system were investigated by using human vascular endothelial cells (HUVEC) and estrogen receptor (ER) positive human breast adenocarcinoma (MCF-7) cells, while the biodistribution and bioelimination of the nanocomposites were investigated by MRI and histological examination in a rat breast cancer model. The contrast of the MR image of tumor tissue was enhanced by
SPION/K-PA. MRI and biodistribution studies confirmed the feasibility of SPION/K-PA for in vivo tumor imaging.

\section{Experimental section}

\section{Materials}

1-Dodecylamine (98\%) and iron(III) 2,4-pentanedionate (97\%) $\left(\mathrm{Fe}(\mathrm{acac})_{3}\right)$ were purchased from Alfa Aesar. 1,2-Hexadecanediol was purchased from Sigma-Aldrich. Lauric acid and trifluoroacetic acid (TFA) were purchased from Merck. Chemicals required for peptide synthesis, such as 9-fluorenylmethoxycarbonyl (Fmoc), tert-butyloxycarbonyl (Boc) protected amino acids, [4-[ $\alpha$ - $\left(2^{\prime}, 4^{\prime}\right.$-dimethoxyphenyl)Fmoc-aminomethyl $]$ phenoxy]-? ?acetamidonorleucyl-MBHA resin (Rink amide MBHA resin) and 2-(1H-benzotriazol-1-yl)-1,1,3,3-tetramethyluronium hexafluoro-? ?phosphate (HBTU) were purchased from NovaBiochem. All reagents and solvents were used as received. Water used during the experiments was deionized by a Millipore Milli-Q purifier system and had a resistance of $18 \mathrm{M} \Omega \mathrm{cm}$.

\section{Synthesis of superparamagnetic iron oxide particles}

Superparamagnetic nanoparticles were synthesized according to a previously described procedure with minor modifications. ${ }^{27}$ Briefly, 6 mmol (1.112 g) lauric acid, 6 mmol (1.2 g) laurylamine and $10 \mathrm{mmol}$ (2.584 g) 1,2-hexadecanediol were mixed in $20 \mathrm{~mL}$ of dibenzylether and heated. When the reaction medium reached $200{ }^{\circ} \mathrm{C}, 2 \mathrm{mmol}(0.706 \mathrm{~g}) \mathrm{Fe}(\mathrm{acac})_{3}$ was added and the sample was stirred for $1-1.5 \mathrm{~h}$ at $200^{\circ} \mathrm{C}$ under inert atmosphere. A condenser was added to the setup for reflux and the system was moved to a hot jacket heater to increase the temperature to $300{ }^{\circ} \mathrm{C}$. Following $1 \mathrm{~h}$ of stirring at this temperature, the mixture was allowed to cool to room temperature and $50 \mathrm{~mL}$ ethanol was added under air. The mixture was centrifuged at $8000 \mathrm{rpm}$ for $15 \mathrm{~min}$, the supernatant was removed and $2-3 \mathrm{~mL}$ of hexane was added to the pellet. After $1 \mathrm{~min}$ of sonication, $20-30 \mathrm{~mL}$ ethanol was added to the mixture. Lastly, the sample was centrifuged again and the brownish supernatant was discarded. Ethanol addition, centrifugation and decantation steps were repeated until the supernatant was observed to exhibit a clean color. The colorless supernatant was then discarded and 6-7 $\mathrm{mL}$ hexane was added to the pellet. Superparamagnetic iron oxide nanoparticles were obtained by sonication for $5 \mathrm{~min}$ followed by centrifugation for $10 \mathrm{~min}$ at $6000 \mathrm{rpm}$, and stored at $4{ }^{\circ} \mathrm{C}$.

\section{Peptide synthesis}

MBHA Rink amide resin was selected as the solid support for the synthesis of the lauryl-PPPGK-Am amphiphilic peptide, and used at a loading of $0.59 \mathrm{mmol} \mathrm{g}^{-1}$. Amino acid couplings were carried out in DMF in the presence of 2 equivalents of Fmoc protected amino acid, 1.95 equivalents of HBTU and 3 equivalents of $N, N$-diisopropylethylamine (DIEA) for $2 \mathrm{~h}$. Base-labile Fmoc protecting groups were removed by treatment with $20 \%$ piperidine/dimethylformamide (DMF) solution for $20 \mathrm{~min}$. Acid-labile groups were cleaved and the peptide was removed from the resin using a cleavage cocktail solution (trifluoroacetic acid (TFA) : triisoproplysilane (TIS) : $\mathrm{H}_{2} \mathrm{O}$ in the ratio of 
$95: 2.5: 2.5$ ), which was added to the beads and left to react for $2 \mathrm{~h}$ at room temperature. Excess TFA was then removed by rotary evaporation, the remaining viscous peptide solution was treated with ice-cold diethyl ether, and the resulting white pellet was freeze-dried. The chemical composition of the amphiphilic peptide was elucidated by reverse phase HPLC using an Agilent 6530 accurate-Mass Q-TOF LC/MS equipped with an Agilent 1200 HPLC. A Phenomenex Luna $3 \mu$ C8 100A $(50 \times 3.00 \mathrm{~mm})$ column and a water $(0.1 \%$ formic acid $)$ /acetonitrile $(0.1 \%$ formic acid) gradient were used as stationary phase and mobile phase, respectively. The corresponding peptide was purified on an Agilent 1200 HPLC having Agilent Zorbax 300SB-C8 (21.2 $\times$ $150 \mathrm{~mm})$ column with a water $(0.1 \%$ TFA $) /$ acetonitrile $(0.1 \%$ TFA) gradient.

\section{Surface coating of SPIONs}

$50 \mathrm{mg}$ of lauryl-PPPGK-Am amphiphilic peptide molecule was dissolved in deionized water $(20 \mathrm{~mL})$, heated up to $55-65{ }^{\circ} \mathrm{C}$ and ultrasonicated. $7 \mathrm{mg}$ of SPION nanoparticles in hexane $(2 \mathrm{~mL})$ was then added to the peptide solution during the sonication process. Amphiphilic peptide molecules and SPIONs were mixed at a weight ratio of $7: 1$ and a volume ratio of $10: 1$, respectively. After $1 \mathrm{~h}$ of repeated sonication and vortexing steps at indicated temperature range, the solution was purified by centrifugation at $6500 \mathrm{rpm}$ for $5 \mathrm{~min}$, using ultrafiltration tubes with $50 \mathrm{kDa}$ cut-off membranes (Millipore Amicon UltraRegenerated Cellulose). This step was repeated at least 2 times.

\section{Characterization of SPIONs and hybrid SPION-peptide system}

Morphological characterization. TEM was performed using a FEI Tecnai G2 F30. $10 \mu \mathrm{L}$ of 50-fold diluted, PA-functionalized and non-functionalized SPION samples were placed on a carbon film (300 mesh) coated copper grid and incubated for $5 \mathrm{~min}$. The excess solution was then removed by pipetting, and the grids were dried under fume hood at room temperature for over 2 h. Selected area electron diffraction (SAED) patterns of hydrophobic SPIONs were taken at an accelerating voltage of $200 \mathrm{kV}$ by TEM. Nanoparticle diameters were determined by using Image $\mathrm{J}$ software from at least 100 nanoparticles.

X-ray diffractometry analysis. Crystal structures of magnetite nanoparticles were investigated by a PANalytical X'Pert Powder Diffractometer operating at $45 \mathrm{kV}$ and $40 \mathrm{~mA}$. Analyses were performed under $\mathrm{Cu} \mathrm{K} \alpha$ radiation $(\lambda=0.15418 \mathrm{~nm})$ in the range of $2 \theta=10-80^{\circ}$. Lyophilized powders were grinded before the analysis and dispersed homogeneously into the holder. All XRD patterns were smoothed and backgrounds were subtracted by using instrument's software (X'Pert High Score).

Chemical characterization by infrared spectroscopy. FTIR analysis was carried out using a Bruker VERTEX 70 with Hyperion scanning to confirm the presence of both iron oxide nanoparticles and the organic coating layer. Prior to the pellet preparation, all samples were lyophilized or dried in a vacuum oven (Memmert GmbH + Co. KG, Germany). SPION, SPION/KPA and K-PA samples were then mixed with $\mathrm{KBr}(1: 99$ (w) $\mathrm{w} \%)$ ), pelleted by grinding and analyzed at a wavelength range of $400-4000 \mathrm{~cm}^{-1}$.
Thermogravimetric analysis. Thermal gravimetric analyses (TGA) were performed using Perkin-Elmer TGA to analyze the organic/inorganic composition of SPION/K-PA samples. Sample temperature was increased from room temperature to $800{ }^{\circ} \mathrm{C}$ with a heating rate of $10{ }^{\circ} \mathrm{C} \mathrm{min}^{-1} .3-5 \mathrm{mg}$ of lyohilized K-PA and SPION/K-PA samples were used for the analysis. Hydrophobic SPIONs dissolved in hexane solutions were dried in a vacuum oven (Memmert $\mathrm{GmbH}+\mathrm{Co}$. KG, Germany) prior to measurement.

Particle size and zeta potential analysis. Hydrodynamic sizes and zeta potentials of the particles were measured by dynamic light scattering (DLS). A Malvern Nanosizer/Zetasizer nano-ZS ZEN 3600 (Malvern Instruments, USA) instrument with a detector angle of $173^{\circ}$ was used for DLS analysis. Hydrophobic particles and hydrophilic particles were diluted with hexane and water, respectively, and measurements were performed in quartz cuvettes. In addition, dip cell electrodes were used in zeta potential measurements. All analyses were measured in triplicate and standard deviations were calculated from the mean of data. For experiments conducted in 5\% dextrose solution at $25{ }^{\circ} \mathrm{C}$, refractive index, viscosity and dielectric constant values were assumed to be $1.34,1.03 \mathrm{~Pa} \mathrm{~s}$ and 77.37, respectively. The concentration of iron oxide nanoparticles was set to $250 \mu \mathrm{g} \mathrm{mL}{ }^{-1}$ for all experiments, and the refractive index of magnetite particles was taken as 2.42 .

Iron content measurement. Iron concentration was determined through inductively coupled plasma-mass spectrometry (ICP-MS, Thermo X Series II) and colorimetric Prussian blue assay prior to PA coating and in vitro and bioimaging experiments. In the former method, a standard curve was derived by dissolving and serially diluting an iron-containing plasma emission standard solution (1000 ppm, VWR-BDH Prolabo) in $2 \%$ nitric acid to obtain 500, 250, 100 and $50 \mathrm{ppb}$ standard iron concentrations. SPION solutions were dissolved in concentrated nitric acid before dilution and heated up to $60^{\circ} \mathrm{C}$ for digestion prior to measurements.

In the Prussian blue assay, a calibration curve was plotted by preparing serially diluted iron solutions (1000 ppm, VWR-BDH Prolabo) in $6 \mathrm{~N} \mathrm{HCl}$. All samples including standards and unknowns were digested at $65^{\circ} \mathrm{C}$ for an hour for the conversion of iron from nanoparticle form to ionic form. $160 \mu \mathrm{L} \mathrm{HCl}$ and 40 $\mu \mathrm{L}$ of $5 \% \mathrm{~K}_{4} \mathrm{Fe}[\mathrm{CN}]_{6}$ was then added to $160 \mu \mathrm{L}$ of samples. Three independent repeats of each sample were prepared for measurement, and $\mathrm{HCl}$ and $5 \% \mathrm{~K}_{4} \mathrm{Fe}[\mathrm{CN}]_{6}$ in water was used as blank solution. Samples were allowed to stain for 20-25 min, and solution color was observed to change from yellow to blue in high-concentration samples following the reaction. UV absorbance was measured at $690 \mathrm{~nm}$ by using Spectramax M5 (Molecular Devices).

Peptide content determination. Peptide-coated SPIONs were purified from excess amphiphilic peptide molecules by ultrafiltration using Millipore Microcon Centrifugal Filter Units at a cut-off point of 50k MW. SPIONs were filtered 2 to 3 times at $6500 \mathrm{rpm}$ for $5 \mathrm{~min}$. The eluted parts were collected at each step and peptide concentrations were measured by NanoDrop 2000 (Thermo Scientific). Results were compared with the results 
obtained from samples prepared with known amounts of PAs. Peptide quantification method offered by Thermo Scientific was utilized to measure the absorbance at analysis wavelength of $205 \mathrm{~nm}$ with an extinction coefficient of $31 \mathrm{mg} \mathrm{mL}^{-1}$ at $1 \mathrm{~cm}$ path length. ${ }^{28,29}$ Peptide solutions were prepared at different concentrations in the range of $0.5 \mathrm{mg} \mathrm{mL}^{-1}$ to $0 \mathrm{mg} \mathrm{mL} \mathrm{m}^{-1}$ by serial dilution.

In vitro studies. Viability profiles of human vascular endothelial cells (HUVEC) and estrogen receptor (ER) positive human breast adenocarcinoma (MCF-7) cells were characterized in the presence of amphiphilic peptides and superparamagnetic iron oxide nanoparticles (SPIONs). HUVECs were kindly donated by Yeditepe University, Istanbul, Turkey. MCF-7 cells were obtained from the American Type Culture Collection (ATCC, Manassas, VA, USA).

Cells were cultured in Dulbecco's Modified Eagle's Medium (DMEM) (Sigma Chemicals, St. Louis, USA) supplemented with $10 \%$ fetal bovine serum (Gibco, Carlsbad, CA, USA) and 1\% penicillin/streptomycin. Culturing was performed in standard humidified incubators with constant $5 \% \mathrm{CO}_{2}$ at $37{ }^{\circ} \mathrm{C}$. Cells were harvested with trypsin-EDTA after reaching $80 \%$ confluency.

Alamar blue assay was used to evaluate the biocompatibility of free K-PA and SPION/K-PA on HUVECs and MCF-7 cells. Harvested cells were seeded into 96-well plates (Costars, Corning Inc., NY, USA) with their respective standard culture media at densities of $1 \times 10^{4}$ cells per well. Sterilized and sonicated free peptides and SPION/K-PA were diluted 1:4 in culture medium prior to use. The final concentration ranges for the PA and nanoparticles were $0-850 \mu \mathrm{M}$ (equivalent content of iron assembled with PA is indicated in the figure) and 0-200 $\mu \mathrm{g}$ $\mathrm{mL}^{-1}$, respectively. After 21 or $45 \mathrm{~h}$ of incubation, $100 \mu \mathrm{L}$ of Alamar blue solution ( $10 \%$ in serum free culture medium) was added to each well after discarding old medium. Following $3 \mathrm{~h}$ of incubation, fluorescence readings of the wells were recorded by an M5 Microplate Reader (Molecular Devices, USA) with excitation at $530 \mathrm{~nm}$ and emission at $590 \mathrm{~nm}$. Biocompatibility of the samples on vascular cells was also evaluated by live-dead assay (Invitrogen), according to the manufacturer's instructions. HUVECs were seeded into 96-well plates with their standard culture medium at a density of $5 \times 10^{3}$ cell per well and treated with SPION/K-PA $\left(75 \mu \mathrm{g} \mathrm{mL}{ }^{-1}\right.$, according to Fe concentration) and K-PA $\left(214 \mu \mathrm{g} \mathrm{mL} \mathrm{mL}^{-1}, 317 \mu \mathrm{M}\right)$ for $24 \mathrm{~h}(n=3)$. HUVECs were then washed with sterile phosphate buffered saline (PBS) and stained with $1 \mu \mathrm{M}$ calcein-AM and $2 \mu \mathrm{M}$ ethidium homodimer-1. Five images were taken under fluorescence microscopy and quantified using Image $\mathrm{J}$ for each sample.

SPION accumulation was visualized by staining cells with Prussian blue, which imparts a blue-purple color in the presence of iron. HUVECs were used for Prussian blue staining. Cells were seeded at a density of $1 \times 10^{4}$ cells per well into 96well plates, incubated until $80 \%$ confluency and subsequently treated with SPION/K-PA $\left(75 \mu \mathrm{g} \mathrm{mL}{ }^{-1}\right)$. After $6 \mathrm{~h}$ of incubation, medium was discarded and unbound particles were removed by washing with PBS $(10 \times)$ three times. The cells were then fixed with $4 \%$ paraformaldehyde $(100 \mu \mathrm{L})$ for approximately $15 \mathrm{~min}$. A mixture of $6 \mathrm{~N} \mathrm{HCl}: 20 \% \mathrm{~K}_{4} \mathrm{Fe}[\mathrm{CN}]_{6}, 1: 1(\mathrm{v} / \mathrm{v})$ was prepared immediately prior to use, and $100 \mu \mathrm{L}$ of solution was added to each well. Cells were then incubated for $60 \mathrm{~min}$, washed with PBS and examined under optical microscope at various magnifications.

In vivo studies. All protocols involving animals were approved by the Institutional Animal Care and Use Committee of Diskapi Yildirim Beyazit Training and Research Hospital. A total of 36 Sprague-Dawley rats (12-14 week old, weighing about 200-250 g) were supplied from ADACELL Laboratories (Ankara, Turkey) and used for MRI experiments and histological studies. Animals were randomly divided into two groups (healthy and tumor-bearing). Animals were maintained at constant temperature and a relative humidity of 50-60\% under $12 \mathrm{~h}$ light/dark cycles, and had access to food and water ad libitum. Mammary carcinomas were induced through subcutaneous injections into the second right mammary pads of adult female rats using single doses of $50 \mathrm{mg} \mathrm{kg}^{-1}$ body weight of 7,12dimethylbenz( $\alpha$ )anthracene (DMBA) dissolved in sesame oil. Rats were palpated weekly to check for tumor formation. Spontaneous mammary tumor formation was observed 3 months after chemical injection. The volume of each tumor was measured by digital caliper and calculated using the following formula: tumor volume $\left(\mathrm{mm}^{3}\right)=\left(D \times d^{2}\right) / 2(D$ : big diameter, $d$ : small diameter of tumor). ${ }^{30}$ MRI measurements were performed when tumor volumes reached $100 \mathrm{~mm} .^{3}$

In order to study the efficiency of SPION/K-PA as a negative contrast agent for MRI, healthy and tumor-bearing rats were randomly divided into 3 groups (4-6) and imaged with or without SPION/K-PA administration. A without-injection group was used as control. In other groups, rats were slowly injected with SPION/K-PA (5 mg Fe per $\mathrm{kg}$ body weight in 5\% dextrose solution) through the tail vein and sacrificed after $4 \mathrm{~h}$ or 15 days. During imaging, Spraque-Dawley rats were anesthetized by the intraperitoneal injection of ketamycin $\left(80 \mathrm{mg} \mathrm{kg}^{-1}\right)$ and xylazine (20 mg kg$\left.{ }^{-1}\right)$. MRI was performed using a $1.5 \mathrm{~T}$ clinical whole body MR scanner (Philips MR system intera) within a sense C3 small surface coil. Images of rats were obtained immediately prior to SPION treatment, during nanoparticle injection, and at $1 \mathrm{~h}, 4 \mathrm{~h}$ and 15 days after injection, with the following sequences and parameters: m-GRASE (gradient and spin-echo weighted imaging): act. TR (ms) 2126, active TE (ms) $n$ $\times 18$, field of view $(\mathrm{cm}) 160 \times 200$, min slice gap $(\mathrm{mm}) 0$, thickness $2.2 \mathrm{~mm}$, ACQ matrix $M \times p(\mathrm{~mm}) 84 \times 72 . T_{2^{-}}$ Weighted spin echo coronal: TR (ms) 3000, TE (ms) 80, field of view $(\mathrm{cm}) 160 \times 200$, slice gap $(\mathrm{mm}) 0$, thickness $2.2 \mathrm{~mm}$, ACQ matrix $M \times p(\mathrm{~mm}) 388 \times 270 . T_{2}$-weighted fat sat: TR $(\mathrm{ms})$ 3000, TE $(\mathrm{ms}) 80$, field of view $(\mathrm{cm}) 160 \times 200$, slice gap $(\mathrm{mm}) 0$, thickness $2.2 \mathrm{~mm}$, ACQ matrix $M \times p(\mathrm{~mm}) 388 \times 270$. All MRI data were transferred to and analyzed on a diagnostic workstation equipped with dedicated software for MRI examination (Philips extended MR workspace 2.6.3.4 2009). Images were evaluated by two radiologists with a minimum of 10 years of experience in the field of body MRI. In order to compare SPIONmediated darkening, all images were collected at the same image depth. Signal intensities (SI) of the regions of interest (ROI) were collected from circles of equivalent sizes $(\sim 5 \mathrm{~mm}$ 
diameter) drawn on the liver, spleen, kidney, breast or tumor tissue. The same procedure was applied to the no-injection group as control. Signal intensity differences were analyzed from all slice images of all tested organs and the breast tumor. Relative signal enhancement was calculated using SI values acquired before (SI pre) and after (SI post) the injection of SPIONs, per the following formula: $1+$ [(SI post - SI pre)/SI pre].

Histological analysis was performed on tissues obtained from the harvested organs, and their general appearances, clearance profiles and tissue distributions were evaluated to further analyze the in vivo biosafety and efficiency of SPION/KPA. Animals were euthanized after the completion of MR imaging. Mammary tumor, liver, spleen, and kidneys were dissected and fixed overnight in $10 \%$ formalin, embedded in paraffin, sectioned with a Leica microtome (5 $\mu \mathrm{m}$ thickness), and stained with hematoxylin and eosin as described elsewhere. ${ }^{31}$ Iron deposits were identified using standard Prussian blue staining protocol. Histological sections were observed under optical microscopy.

Statistical analysis. All data are presented as \pm standard error of mean (SEM) for all in vitro and in vivo results. Plotting was performed using the GraphPad Prism 5 software (La Jolla, CA). Viability results were evaluated using one-way analysis of variance (ANOVA) with Tukey's test for group-wise comparisons. MRI results were compared by two-way ANOVA test. $p$ values less than 0.05 were considered as statistically significant.

\section{Results and discussion}

\section{Synthesis and characterization of the nanoparticles}

Thermal decomposition is an effective method for the production of iron oxide nanoparticles with minimal variance in shape and size. ${ }^{27}$ Although the method results in the formation of water insoluble nanoparticles and requires further modification for the efficient dispersion in the aqueous environment, it provides higher stability and significant control over the size and shape of the nanoparticle compared to co-precipitation, which is another common method for nanoparticle synthesis. ${ }^{32}$ Here, iron oxide $\left(\mathrm{Fe}_{3} \mathrm{O}_{4}\right)$ nanocrystals were synthesized through the high-temperature reaction of iron(III) acetylacetonate, $\mathrm{Fe}(\mathrm{acac})_{3}$, in dibenzyl ether in the presence of 1,2hexadecanediol, lauric acid and dodecylamine. Since the produced nanoparticles were hydrophobic due to their lauric acid and dodecylamine capping, they were dispersed in a nonpolar organic solvent, hexane. Subsequently, they were stored at $4{ }^{\circ} \mathrm{C}$ and used for further modification in the course of six months. The iron oxide nanoparticles, which were prepared via thermal decomposition method, had an average size of 4-16 nm and displayed superparamagnetic characteristics. ${ }^{33}$ In our previous study, this behavior was verified by vibrating sample magnetometer (VSM) measurements. ${ }^{26}$ We furthermore observed that the synthesized superparamagnetic iron oxide nanoparticles (SPIONs) possessed rapid magnetic responsivity to an applied external magnetic field (Fig. S1†). TEM images (Fig. 1c) suggest that monodisperse SPIONs had a narrow size distribution with uniform spherical morphologies. Their average diameter was found to be $5.5 \mathrm{~nm}$, with a standard
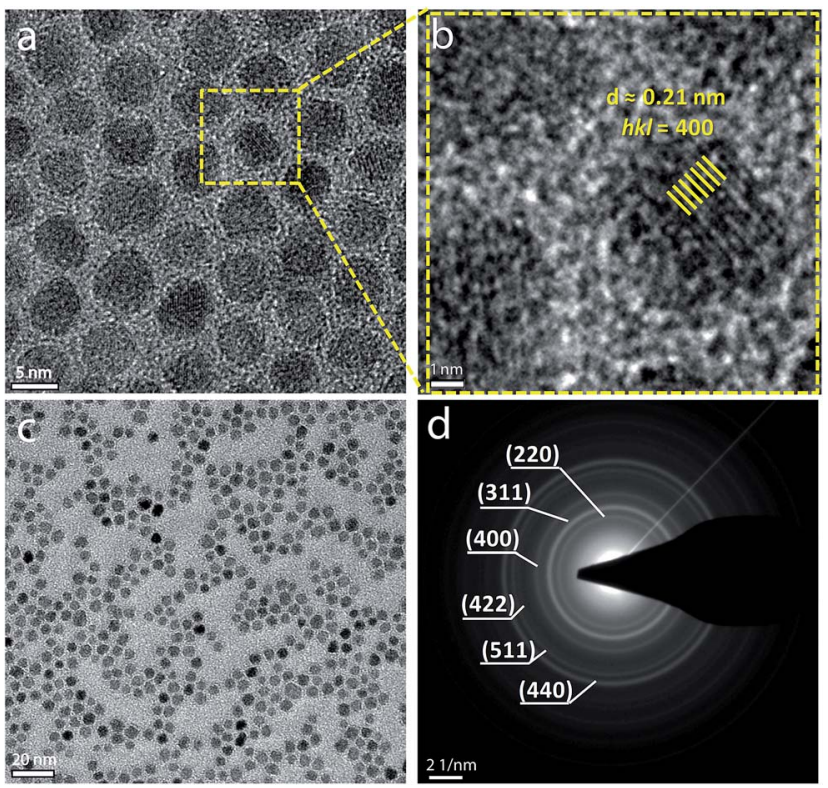

Fig. 1 (a) HR-TEM image of SPIONs, (b) lattice fringe corresponds to the (400) lattice plane of magnetite nanoparticles, (c) TEM image of SPIONs, (d) SAED pattern of SPIONs.

deviation of $0.6 \mathrm{~nm}$. Dynamic light scattering (DLS) analysis was also performed on hexane-dispersed nanoparticles, diameters of which were found to be $7.1 \pm 0.3 \mathrm{~nm}$ and were in agreement with the diameter values obtained from imaging (Fig. S2 $\dagger$ ). Lattice fringes can be observed in the high resolution transmission electron microscopy (HR-TEM) image of SPIONs (Fig. 1a and b), demonstrating the crystallinity of the respective nanoparticles. A specified area was magnified from the image to visualize individual lattice planes to a greater extent. The measured $d$-spacing of $2.12 \AA$ corresponded to that of the (400) plane $(2.09 \AA)$ of magnetite. ${ }^{34}$ Additionally, the circular rings present in the electron diffraction pattern confirmed the polycrystalline nature of the magnetite nanoparticles (Fig. 1d). Lattice spacings corresponding to each ring were calculated based on the diffraction patterns and showed good agreement with that of magnetite (JCPDS, no. 19-0629). XRD analysis result of SPIONs was also consistent with these obtained from SAED patterns and the literature (Fig. 3a). ${ }^{35}$ The diffraction patterns demonstrated in Fig. 3 a were used to estimate the $d$-spacings of SPIONs by using Bragg's law. The calculated values along with the respective Miller indices values $(h k l)$ and reference data are listed in Table S1.† Results acquired from these techniques supported each other and revealed that the synthesized SPIONs had an inverse spinel structure. ${ }^{34}$

A variety of methods are available to increase the watersolubility and enhance the dispersion stability of SPIONs in physiological conditions, the optimization of which is critical for the in vivo applicability of nanoparticles. Principal among these methods are the ligand addition or ligand exchange approaches. ${ }^{36}$ In the former method, noncovalent interactions, such as electrostatic interactions, hydrophobic interactions and hydrogen bonding, are the major driving forces for the physical adsorption of molecules to the surface of SPIONs. Amphiphilic 
a)

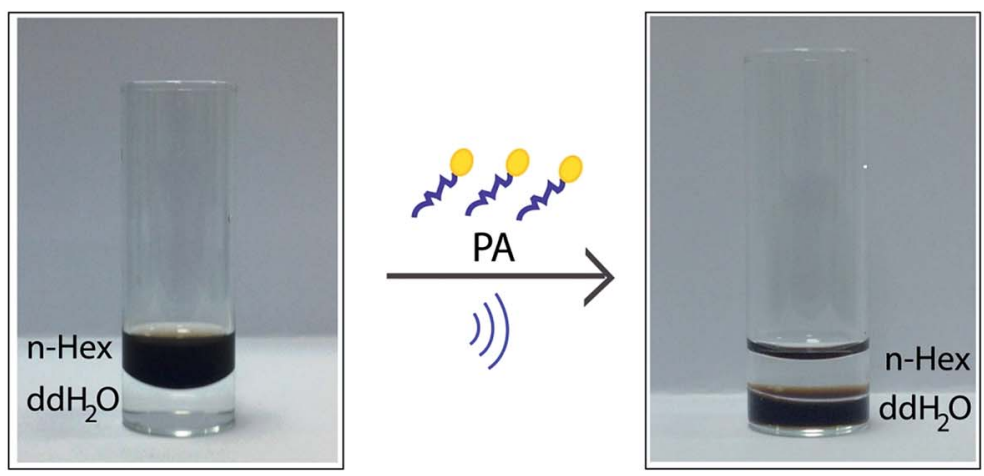

b)

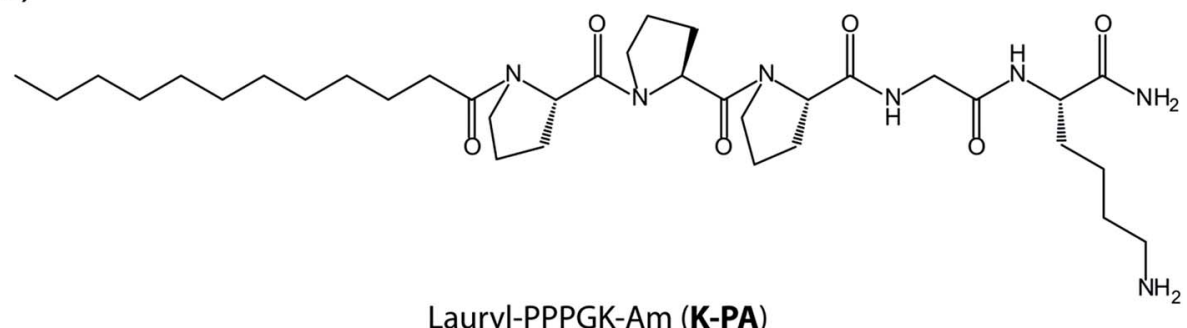

Fig. 2 (a) Photograph of modification of hydrophobic SPIONs with peptide molecules, (b) chemical structure of the peptide molecule, K-PA.

macromolecular ligands and liposomes have been used for the preparation of water-dispersible SPIO nanocomposites. ${ }^{19,37}$ In this study, to improve the biocompatibility and biodegradability of the functionalized SPIONs, PA molecules were utilized in the production of SPION/PA nanocomposites. In the amphiphilic peptide design, three main segments were used for the development of stable, well-dispersed SPION/PA system. While alkyl tail of peptide enhancing the hydrophobic interactions between alkylated surface of SPIONs and peptide, the proline-rich segment (-PPP) with its hydrogen bond breaking property improves intermolecular cohesive interactions by avoiding nanofiber formation, and lastly charged amino acid residue is used to solubilize the system as well as to prevent its aggregation. Proline-rich amphiphilic peptides are known to exhibit random coil conformations, as analyzed by circular dichroism..$^{38,39}$ The PA was synthesized by solid phase peptide synthesis strategy based on orthogonal protection/deprotection chemistry, and its chemical structure was verified by liquid chromatography and mass spectroscopy (Fig. S3†). The change in the design led to an increase in the integration of the lauryl group of the PA into the alkylated surface of SPIONs, resulting in enhanced solubility in aqueous environment. The water stabilization of SPIONs was achieved by simply sonicating two immiscible solutions at the temperature range of $55-65{ }^{\circ} \mathrm{C}$, below the boiling point of hexane. While SPIONs transferred into the water phase, hexane slowly evaporated with time. After the purification steps, the nanoparticles were concentrated through the ultrafiltration method. As shown in Fig. 2, SPIONs were successively transferred into the aqueous environment with this method. The eluted parts were also collected to indirectly determine the amount of peptide in the SPION/K-PA coassembled system. Excess peptide amount was calculated by measuring peptide bond absorption at $205 \mathrm{~nm} .{ }^{28}$ Since PA molecules were not purely composed of amino acids but also contained an alkylated "tail" region, we primarily normalized the values by measuring known amount of peptide samples prepared at different concentrations. After normalization, the value was subtracted from the initial peptide amount used for the SPION functionalization. According to the calculations, nearly $35 \%$ of initial peptide remained in the co-assembled system. SPION/K-PA can be stored at high concentrations in the refrigerator for 4-5 months without losing its water dispersibility and magnetic responsivity.

The SPION/K-PA system was further examined by using a variety of material characterization techniques. The XRD pattern of pure amphiphilic peptides showed a diffused halo peak registered between 10 and $30^{\circ}(2 \theta)$, revealing the amorphous nature and confirming the disorganized structure of PAs (Fig. 3a). The SPION/PA nanocomposite exhibited the same diffraction pattern as those obtained before PA functionalization, indicating that SPIONs retained their crystal structure after being assembled with PAs. FTIR spectra of non-bound PAs, SPIONs and their co-assembled form are presented in Fig. 3b. Hydrophobic SPIONs showed two prominent bands at 596 and $438 \mathrm{~cm}^{-1}$ which were attributed to the $\mathrm{Fe}-\mathrm{O}$ vibrations of the ferrite core. ${ }^{40}$ The peaks at 2926 and $2848 \mathrm{~cm}^{-1}$ were attributed to the asymmetric and symmetric stretching vibrations of $-\mathrm{CH}_{2}$ groups present in lauric acid and dodecyl amine. Two peaks were observed at 1526 and $1413 \mathrm{~cm}^{-1}$, corresponding to two carboxylate stretches that may be related to the chemisorption 
a)

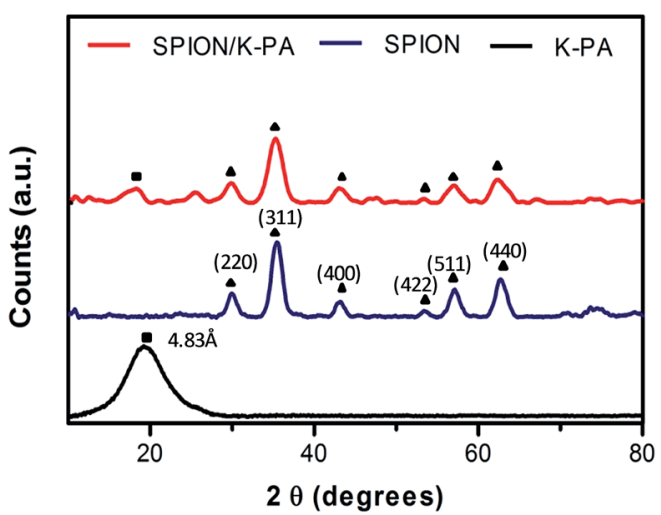

b)

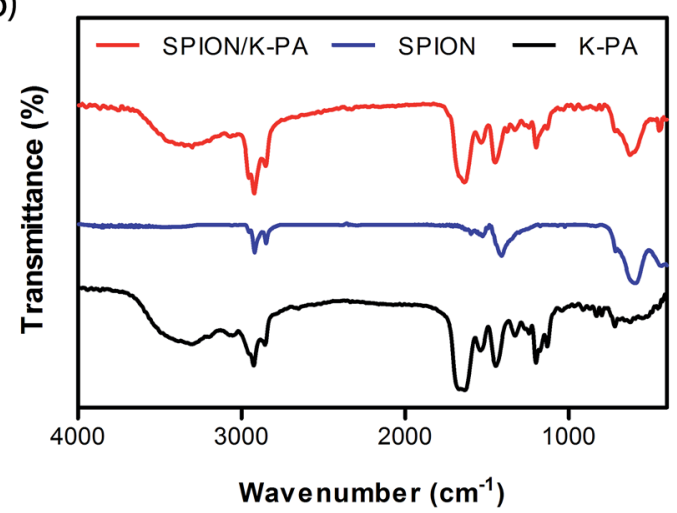

Fig. 3 (a) XRD patterns and (b) FTIR spectra of bare and modified nanoparticles.

of lauric acids on the surface of nanoparticles. In the case of PA molecules, characteristic $\mathrm{C}=\mathrm{O}$ stretching vibrations were observed between 1600 and $1700 \mathrm{~cm}^{-1}$, while $\mathrm{N}-\mathrm{H}$ bending vibrations were found to generate a broad band at 3200-3600 $\mathrm{cm}^{-1}$. The IR spectrum of K-PA also exhibited C-N stretching and $\mathrm{N}-\mathrm{H}$ bending vibration peaks at around $1534 \mathrm{~cm}^{-1}$ corresponding to the amide II band of PA. In the co-assembled form, the previously identified peaks of SPION and PA were both present. Additionally, the strength of $-\mathrm{CH}_{2}$ stretching at 2926 and $2848 \mathrm{~cm}^{-1}$ increased with the integration of the alkyl tail of peptide into the system.

TGA thermograms were obtained to determine the organic/ inorganic ratio of SPION/PA system and the corresponding decomposition temperatures. SPIONs showed two major transitions prior to PA functionalization, with weight losses occurring between $200-450{ }^{\circ} \mathrm{C}$ and $600-750{ }^{\circ} \mathrm{C}$. These losses most likely resulted from the decomposition of the organic surface layer and phase transition from $\mathrm{Fe}_{3} \mathrm{O}_{4}$ to $\mathrm{FeO}$ followed by the deoxidation of $\mathrm{FeO}$, respectively (Fig. S4a †). ${ }^{41}$ In the case of $\mathrm{K}$ PA, the first pronounced mass loss was observed at $220{ }^{\circ} \mathrm{C}$ due to the decomposition of the alkyl tail, and a second mass loss between 300 and $450{ }^{\circ} \mathrm{C}$ was ascribed to the cleavage of peptide bonds and amino acid side groups. After PA functionalization, similar decomposition processes were observed, and a total mass of $52 \%$ was found to be attributable to PAs (Fig.$\mathrm{S} 4 \mathrm{~b} \dagger$ ). Results acquired from TGA analysis also corroborated with the results obtained from the colorimetric PA determination analysis. As shown in Fig. S5, $\dagger$ the peptide functionalization process did not change the shape of the nanoparticles, which were randomly distributed on the TEM grid except for very few overlapping areas. Since there was no staining on the samples, only the core was imaged and the mean diameter of the SPION/K-PA nanoparticles was estimated as $5.3 \pm 0.7 \mathrm{~nm}$, revealing that functionalization did not cause any change in the morphology of nanoparticles in terms of shape or size. When the hydrodynamic size of SPION/K-PA was determined in two different media, water and 5\% dextrose solution, nanoparticles were found to have hydrodynamic sizes of $58.0 \pm 0.7 \mathrm{~nm}$ and $58.6 \pm 0.1 \mathrm{~nm}$ in water and $5 \%$ dextrose, respectively, which are in the range of optimal values for long circulation times in vivo (Fig. S6 and Table S2 $\dagger$ ). ${ }^{42}$ The hydrodynamic sizes did not significantly change over the measured period of time for both solutions, and the photographs of nanoparticle dispersions indicated that they maintained their dispersion stabilities even after a week (Fig. S6†). Nanoparticles dispersed in 5\% dextrose solution exhibited smaller changes in their hydrodynamic sizes compared to those dispersed in water, which is suitable for the storage of these nanoparticles prior to their administration. Their zeta potentials were also measured and found to be positively charged due to the lysine residue present in the PA structure.

\section{In vitro cytotoxicity and uptake studies}

The biocompatibility of SPION/K-PA was evaluated on vascular endothelial cells and human breast cancer cells by using Alamar blue assay. Iron concentrations were adjusted by measuring iron contents of the solutions using two different techniques, inductively coupled plasma-mass spectrometry and colorimetric Prussian blue assay. To determine iron content via colorimetric method, a calibration curve was constructed for solutions prepared from serially diluted reference solutions (Fig. S7 $\dagger$ ), and PA amounts in corresponding SPION/PA solutions were spectroscopically determined. We compared the cytocompatibility profiles of K-PA and SPION/K-PA to understand whether they affect the viability of tumor cells or nontumor cells (Fig. S8†). SPION/K-PA was found to be biocompatible for both HUVECs and MCF-7 cells after $24 \mathrm{~h}$ and $48 \mathrm{~h}$ exposure. In the literature, dextran or citric acid coated nanoparticles were shown to demonstrate higher cytotoxicity on HUVECs at nanomolar concentrations after $24 \mathrm{~h}$ of exposure. ${ }^{43}$ When compared to those nanoparticles, SPION/K-PA is safer for biomedical applications at tested concentrations (Fig. S8†). On the other hand, cytotoxicity assay results also showed that K-PA alone affects cell viabilities in a dose-dependent manner which may result from the presence of positively-charged side chains on the surfaces of unbound peptides that may increase their interactions with the cell membrane, resulting in membrane damage. ${ }^{44}$

Prior to in vivo studies, the biocompatibility of the nanoparticles was further analyzed using live-dead assay. For in vitro studies, selected concentration $\left(75 \mu \mathrm{g} \mathrm{mL} \mathrm{m}^{-1}\right)$ is equivalent of MR administration dosage which was calculated according to the total circulating blood volume of a rat $\left(64 \mathrm{~mL} \mathrm{~kg}^{-1}\right.$ of body 
weight). ${ }^{45}$ The data in Fig. $4 \mathrm{a}$ and $\mathrm{b}$ indicate that there was no considerable reduction in vascular cell viability after exposure to K-PA and SPION/K-PA compared to the no-treatment group. More than $90 \%$ of the cells survived in the presence of SPION/KPA after $24 \mathrm{~h}$ of incubation, suggesting that these nanoparticles could be employed safely in MRI.

The cellular uptake of nanoparticles by endothelial cells was visualized by Prussian blue staining. Clustered blue particles were present on the surfaces and interiors of HUVECs exposed to SPION/K-PA (Fig. 4c). Nanoparticles were retained in cells within $6 \mathrm{~h}$. The binding efficiency of SPION/K-PA reached approximately $90 \%$ for HUVECs. Under the same conditions, no blue dots were observed in non-treated control cells. The strong binding interaction exhibited by SPION/K-PA may result from the electrostatic interactions between the positively charged nanoparticles and the cells, which possess a negatively charged phospholipid membrane. It is known that positively charged nanoparticles have higher uptake rates than negatively charged particles. $^{\mathbf{4 6}}$

\section{In vivo magnetic resonance imaging studies}

We evaluated the capacity of the SPION/K-PA to serve as a negative contrast agent in $T_{2}$-weighted MRI in vivo by using chemically induced model of breast cancer. DMBA is a well known carcinogen which leads to the release of lipid peroxides and formation of free radicals to induce tumorigenesis. ${ }^{47}$

a)

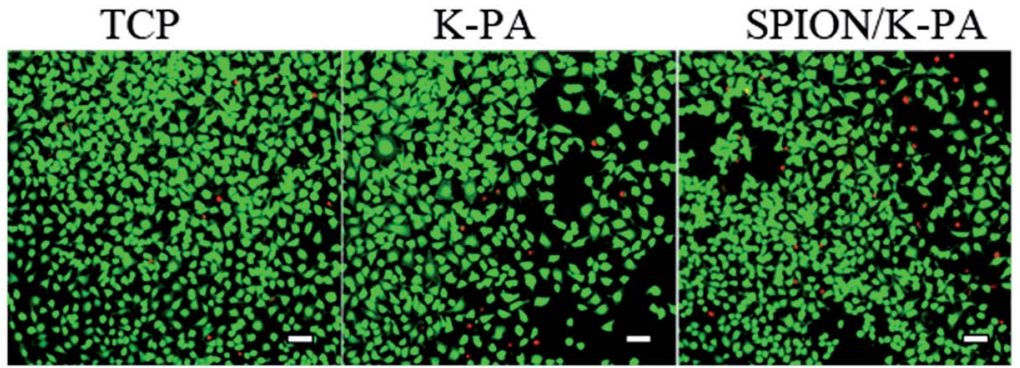

b)

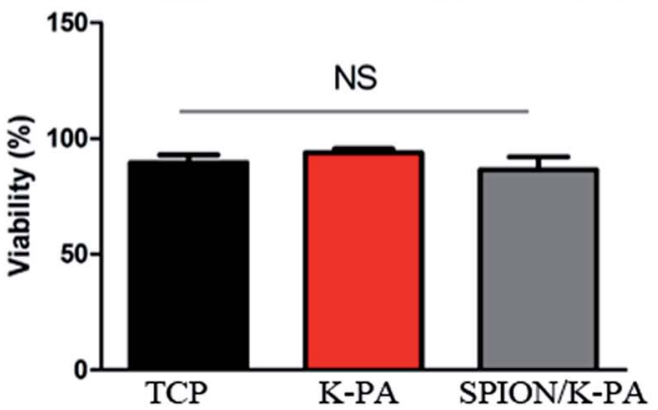

c)

CONTROL

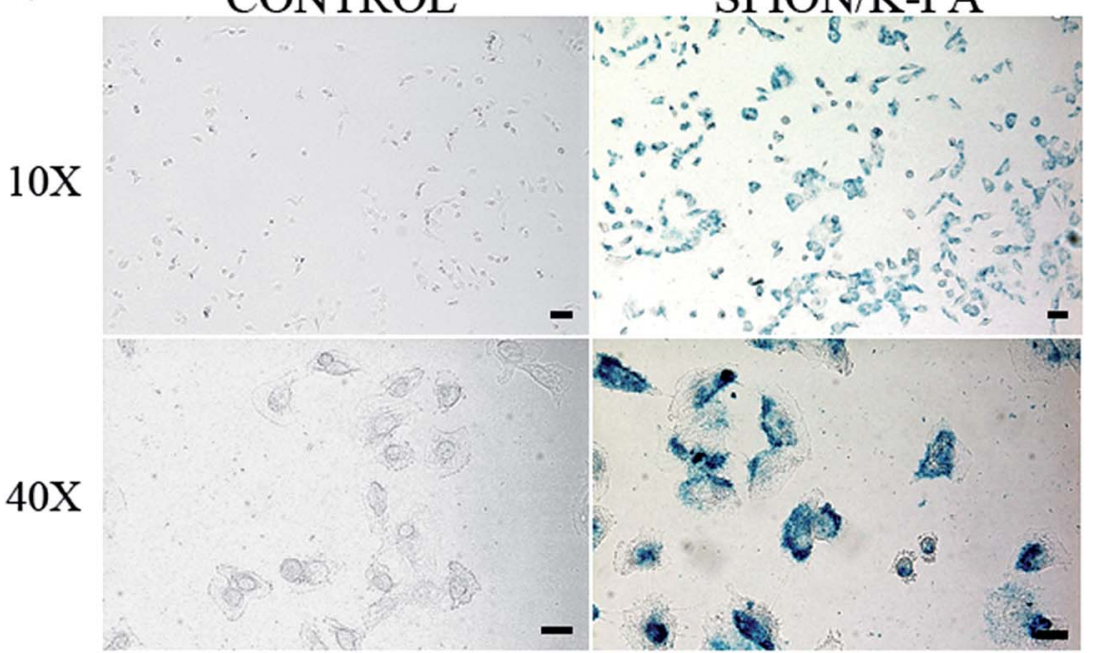

Fig. 4 Viability and Prussian blue staining of HUVECs. (a) Cells were tested for biocompatibility of SPIONs after treatment with K-PA (855 $\mu$ g $\mathrm{mL}^{-1}$ ) and SPION/K-PA (75 $\mathrm{g} \mathrm{mL}^{-1}$ ) for $24 \mathrm{~h}$ by live-dead assay. Live cells were stained with calcein-Am (green) and dead cells were stained with ethidium homodimer-1 (red), scale bar: $100 \mu \mathrm{m}$, magnification: 100×. (b) Quantification of viability of experimental groups. (c) Light microscopy of HUVECs following $6 \mathrm{~h}$ of incubation with and without SPION/K-PA at different magnifications $(100 \times$ and $400 \times)$. Scale bars: $50 \mu \mathrm{m}$ and $20 \mu \mathrm{m}$, respectively. Experiments were performed two independent times in triplicate. TCP: tissue culture plate, NS: non-significant. 
Palpable mammary tumors were developed in DMBA treated animals after 12 weeks. Relative signal intensity (SI) of nanoparticles in tumors, healthy breast tissue and RES organs were determined as a function of time for up to 15 days after contrast agent injection through the tail vein, using a clinical $1.5 \mathrm{~T}$ MRI system with a $T_{2}$-weighed m-GRASE sequence.

MRI results showed that the nanoparticles preferentially accumulated in tumor tissue rather than RES organs. Significant negative enhancement of the MRI signal for SPION/K-PA in the tumor tissue was observed from $1 \mathrm{~h}$ to $4 \mathrm{~h}$, compared to the $T_{2}$-weighted images prior to the administration of SPION/K-PA and non-injected control groups (Fig. 5a). Signal intensity changes resulting from nanoparticle uptake into tumor and other organs were calculated in the region of interest (RIO) of the $T_{2}$-weighted m-GRASE images as a function of time in order to quantify signal contrast enhancement (Fig. 5b). Importantly, signal intensities of mammary tumors decreased by approximately $45 \%$ and $65 \%$ at $1 \mathrm{~h}$ and $4 \mathrm{~h}$ after SPION/K-PA injection, respectively. These results confirmed that SPION/K-PA could

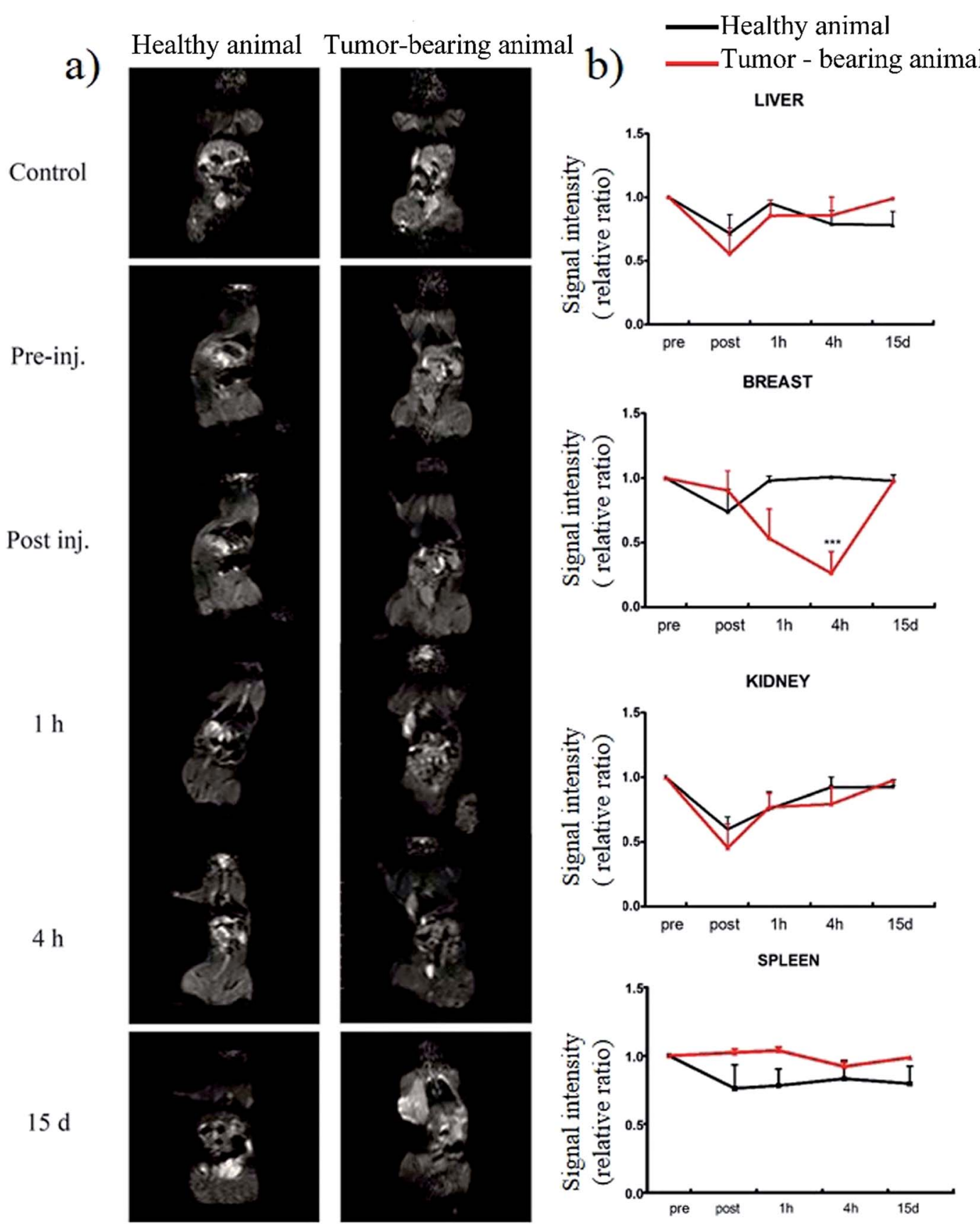

Fig. 5 In vivo MRI. Signal intensity changes in $T_{2}$ relaxations at pre-, immediately after injection (post), $1 \mathrm{~h}, 4 \mathrm{~h}$ and $15 \mathrm{~d}$ post administration of SPION/K-PA $\left(5 \mathrm{mg} \mathrm{kg}^{-1}\right)$ in breast, liver, spleen, kidney and breast tumor of rats. Animals without nanoparticle injection is used as control. A clear gradual signal intensity drop in the breast tumor was observed after SPION/K-PA administration. 


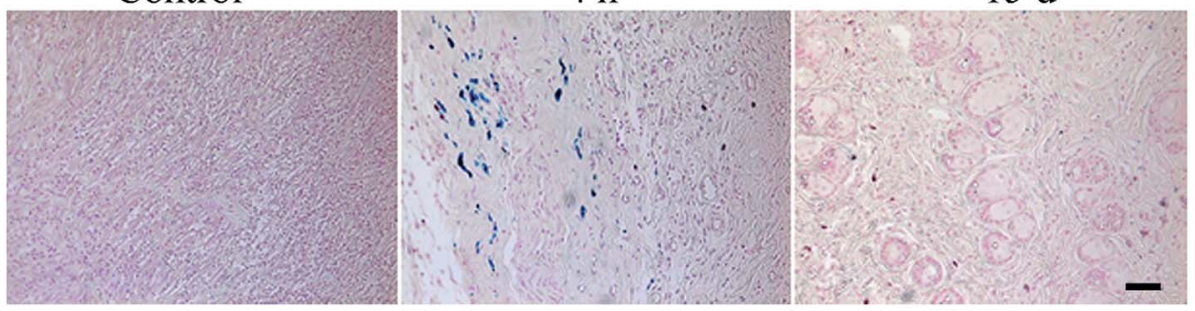

Fig. 6 Prussian blue staining for iron accumulation in the tumor of rats (control) prior to or $4 \mathrm{~h}$ and 15 days following the injection of SPION/K-PA $\left(5 \mathrm{mg} \mathrm{kg}^{-1}\right.$ ) (original magnification, 200x, scale bar represents $50 \mu \mathrm{m}$ ).

serve as an effective negative contrast agent for the diagnosis of cancer by MRI. Additionally, there was no statistically considerable difference between the organs of healthy and tumorbearing animals in terms of the signal drop in breast and RES organs following the administration of nanoparticles. Relative SIs were observed to be reduced in the liver, kidney and spleen of both healthy and tumor-bearing animals immediately following nanoparticle administration, which may be a consequence of the fast blood flow rate in these organs. ${ }^{48}$ Nevertheless, the signal intensity of magnetic nanoparticles in RES organs started to increase to the same levels as the pre-injection groups at $1 \mathrm{~h}$ (Fig. 5b). Compared to the $T_{2}$ values measured using pre-contrast images, the particles did not produce a $T_{2}$ shortening after 15 days in any organ. This indicated that nanoparticles were removed from the organs within two weeks.

Though SPION/PA does not possess any targeting ligands on its surface, it can efficiently accumulate in tumors because of the leaky vascular structure of tumor tissue, which is referred to enhanced permeation and retention (EPR) effect. Using this phenomenon, SPIONs have been previously utilized for in vivo cancer imaging via MRI in the absence of a biologically active targeting ligand. ${ }^{15,49}$ However, there is a distinct lack of information regarding the elimination routes and times of retention associated with SPIONs in different organs. We further studied the accumulation and retention of SPIONs in different organs by using histological analyses.

\section{Histological examination and tissue distribution of SPION/K-} PA

We examined the short and long-term distribution of SPION/ K-PA within the tissue sections of rats after nanoparticle injection. RES organs are usually the main site of accumulation for nanoparticles. Nanoparticle aggregation at these locales may cause inflammation, release of toxic byproducts by biodegradation or uncontrolled and unpredictable patterns of localization. In addition, prolonged nanoparticle exposure may result in hematopoietic, bone marrow and/or renal toxicity. ${ }^{50}$ In particular, the infiltration of monocytes and lymphocytes through the portal space or centrolobular veins is the most important indicator of the inflammatory process in liver. ${ }^{51}$ There was no obvious pathological changes and any lesions in the liver and the number of lymphocytes did not alter in the spleen of both healthy and tumor-bearing rats after nanoparticle injection (Fig. S9 and S10†). Additionally, shortened tube cavity, atrophy of the renal tubular and glomerular epithelial cells, and transparent tubes were not observed in the kidney of experimental groups. These results demonstrated that exposure to SPION/K-PA does not result in inflammation or systemic toxicity not only in short term but also at two weeks post-injection.

To further verify the accumulation and distribution of SPION/K-PA in tumors and harvested organs, Prussian blue staining was carried out as shown in Fig. 6, S11 and S12.† We detected a large number of blue dots in the tumors of rats administered with SPION/K-PA, which indicates the elevated uptake of nanoparticles in tumor tissue. Prussian blue staining of liver, spleen and kidney tissues in the SPION/K-PA group revealed no significant uptake of nanoparticles in both healthy and cancerous rats after $4 \mathrm{~h}$. These results are in accordance with those we obtained by MRI.

When designing an effective nanomaterial for diagnostic applications, one of the most important issues is to increase the material's residence time in the organ of interest and thereby allow imaging to be performed for as long as possible prior to its clearance. However, the material should also be broken down and removed from the body after imaging to prevent its cumulative accumulation. ${ }^{52}$ Taken together, our results demonstrated that SPION/K-PA is biocompatible and biodegradable, while localizing into the tumor site and allowing enhanced MRI imaging following injection. ${ }^{53}$

\section{Conclusion}

In summary, we show peptide coated iron oxide nanoparticles in aqueous environment. The MRI signal of breast tumor tissue was significantly enhanced after administration of the SPION/KPA, which shows that the nanocomposite system is promising as a negative MRI contrast agent. The in vivo observation of SPION/K-PA suggest that the co-assembled system is biocompatible and biodegradable, as confirmed by the long-term consistency of the MRI signal and the material's lack of accumulation in RES organs in histological analyses. Due to the ease of functionalization of the nanoparticles, the practical use of peptide functionalized SPIONs can be further extended by conjugating bioactive epitopes into the peptide construct to selectively target a specific type of receptor or cell. 


\section{Conflict of interest}

The authors have declared that no conflict of interest exists regarding this paper.

\section{Acknowledgements}

A. O. and M. S. E. are supported by TUBITAK BIDEB $2211 \mathrm{PhD}$ fellowship. We would like to thank M. Guler for TEM imaging, E. D. Eren for XRD measurements, E. Yurdakul for his help during MRI, I. Ulusoy for animal experiments and A. D. Ozkan for fruitful scientific discussion.

\section{References}

1 K. K. Shung, M. Smith and B. M. Tsui, Principles of medical imaging, Academic Press, 2012.

2 M. Sardan, A. D. Ozkan, A. Zengin, A. B. Tekinay and M. O. Guler, Therapeutic Nanomaterials, 2016, pp. 197-234.

3 R. W. Brown, Y.-C. N. Cheng, E. M. Haacke, M. R. Thompson and R. Venkatesan, Magnetic resonance imaging: physical principles and sequence design, John Wiley \& Sons, 2014.

$4 \mathrm{H}$. Ammari, An introduction to mathematics of emerging biomedical imaging, Springer, 2008.

5 W. Mulder, G. J. Strijkers, G. Van Tilborg, A. W. Griffioen and K. Nicolay, NMR Biomed., 2006, 19, 142-164.

6 H. Wang and X. Chen, Expert Opin. Drug Delivery, 2009, 6(7), 745-768.

7 A. Bertin, J. Steibel, A.-I. Michou-Gallani, J.-L. Gallani and D. Felder-Flesch, Bioconjugate Chem., 2009, 20, 760-767.

8 M. K. Yu, Y. Y. Jeong, J. Park, S. Park, J. W. Kim, J. J. Min, K. Kim and S. Jon, Angew. Chem., Int. Ed., 2008, 47, 53625365.

9 A. K. Gupta and M. Gupta, Biomaterials, 2005, 26, 3995-4021.

10 Y. w. Jun, J. H. Lee and J. Cheon, Angew. Chem., Int. Ed., 2008, 47, 5122-5135.

11 X. H. Peng, X. Qian, H. Mao, A. Y. Wang, Z. G. Chen, S. Nie and D. M. Shin, Int. J. Nanomed., 2008, 3, 311-321.

12 M. E. Akerman, W. C. Chan, P. Laakkonen, S. N. Bhatia and E. Ruoslahti, Proc. Natl. Acad. Sci. U. S. A., 2002, 99, 1261712621.

13 D. Ling and T. Hyeon, Small, 2013, 9, 1450-1466.

14 M. V. Yezhelyev, X. Gao, Y. Xing, A. Al-Hajj, S. Nie and R. M. O'Regan, Lancet Oncol., 2006, 7, 657-667.

15 N. Lee, Y. Choi, Y. Lee, M. Park, W. K. Moon, S. H. Choi and T. Hyeon, Nano Lett., 2012, 12, 3127-3131.

16 S. J. Lee, H. J. Lee, M. J. Moon, H. Vu-Quang, M. Muthiah, H. L. Che, S. U. Heo, H. J. Jeong, Y. Y. Jeong and I. K. Park, J. Nanosci. Nanotechnol., 2011, 11, 7057-7060.

17 P. Caravan, Acc. Chem. Res., 2009, 42, 851-862.

18 A. J. Cole, V. C. Yang and A. E. David, Trends Biotechnol., 2011, 29, 323-332.

19 D. Ling and T. Hyeon, Small, 2013, 9, 1450-1466.

20 S. Cavalli, D. Carbajo, M. Acosta, S. Lope-Piedrafita, A. P. Candiota, C. Arús, M. Royo and F. Albericio, Chem. Commun., 2012, 48, 5322-5324.
21 J. Xie, K. Chen, H.-Y. Lee, C. Xu, A. R. Hsu, S. Peng, X. Chen and S. Sun, J. Am. Chem. Soc., 2008, 130, 7542-7543.

22 C. G. Hadjipanayis, R. Machaidze, M. Kaluzova, L. Wang, A. J. Schuette, H. Chen, X. Wu and H. Mao, Cancer Res., 2010, 70, 6303-6312.

23 K. J. Landmark, S. DiMaggio, J. Ward, C. Kelly, S. Vogt, S. Hong, A. Kotlyar, A. Myc, T. P. Thomas and J. E. PennerHahn, ACS Nano, 2008, 2, 773-783.

24 C. M. Lee, H. J. Jeong, E. M. Kim, D. W. Kim, S. T. Lim, H. T. Kim, I. K. Park, Y. Y. Jeong, J. W. Kim and M. H. Sohn, Magn. Reson. Med., 2009, 62, 1440-1446.

25 J. Raczkowska, M. Ohar, Y. Stetsyshyn, J. Zemła, K. Awsiuk, J. Rysz, K. Fornal, A. Bernasik, H. Ohar and S. Fedorova, Colloids Surf., B, 2014, 118, 270-279.

26 S. Sulek, B. Mammadov, D. I. Mahcicek, H. Sozeri, E. Atalar, A. B. Tekinay and M. O. Guler, J. Mater. Chem., 2011, 21, 15157-15162.

27 S. Sun and H. Zeng, J. Am. Chem. Soc., 2002, 124, 8204-8205. 28 R. Scopes, Anal. Biochem., 1974, 59, 277-282.

29 M. H. Simonian, Current Protocols in Toxicology, 2004, A. 3G. 1-A. 3G. p. 7.

30 A. C. Obenauf, Y. Zou, A. L. Ji, S. Vanharanta, W. Shu, H. Shi, X. Kong, M. C. Bosenberg, T. Wiesner, N. Rosen, R. S. Lo and J. Massague, Nature, 2015, 520, 368-372.

31 M. K. Stewart, J. F. Bechberger, I. Welch, C. C. Naus and D. W. Laird, Oncotarget, 2015, 6(35), 37185-37199.

32 S. Laurent, D. Forge, M. Port, A. Roch, C. Robic, L. Vander Elst and R. N. Muller, Chem. Rev., 2008, 108, 2064-2110.

33 S. Sun, H. Zeng, D. B. Robinson, S. Raoux, P. M. Rice, S. X. Wang and G. Li, J. Am. Chem. Soc., 2004, 126, 273-279.

34 R. Cornell and U. Schwertmann, The Iron Oxides: Structure, Properties, Reactions, Occurences and Uses, 2nd edn, 1996, pp. 297-344.

35 Z. Li, L. Wei, M. Gao and H. Lei, Adv. Mater., 2005, 17, 10011005.

36 M. Muthiah, I.-K. Park and C.-S. Cho, Biotechnol. Adv., 2013, 31, 1224-1236.

37 S. German, N. Navolokin, N. Kuznetsova, V. Zuev, O. Inozemtseva, A. Anis'kov, E. Volkova, A. Bucharskaya, G. Maslyakova and R. Fakhrullin, Colloids Surf., B, 2015, 135, 109-115.

38 M. O. Guler, R. C. Claussen and S. I. Stupp, J. Mater. Chem., 2005, 15, 4507-4512.

39 D. Mumcuoglu, M. Sardan, T. Tekinay, M. O. Guler and A. B. Tekinay, Mol. Pharmaceutics, 2015, 12, 1584-1591.

40 M. Ma, Y. Zhang, W. Yu, H.-y. Shen, H.-q. Zhang and N. Gu, Colloids Surf., A, 2003, 212, 219-226.

41 M. Mahdavi, M. B. Ahmad, M. J. Haron, F. Namvar, B. Nadi, M. Z. A. Rahman and J. Amin, Molecules, 2013, 18, 75337548.

42 C. Boyer, M. R. Whittaker, V. Bulmus, J. Liu and T. P. Davis, NPG Asia Mater., 2010, 2, 23-30.

$43 \mathrm{X} . \mathrm{Wu}, \mathrm{Y}$. Tan, H. Mao and M. Zhang, Int. J. Nanomed., 2010, 5, 385-399.

44 D. Gaspar, A. S. Veiga, C. Sinthuvanich, J. P. Schneider and M. A. R. B. Castanho, Biochemistry, 2012, 51, 6263-6265. 
45 K. H. Diehl, R. Hull, D. Morton, R. Pfister, Y. Rabemampianina, D. Smith, J. M. Vidal and C. van de Vorstenbosch, J. Appl. Toxicol., 2001, 21(1), 15-23.

46 C. Yang, J. Uertz, D. Yohan and B. D. Chithrani, Nanoscale, 2014, 6, 12026-12033.

47 K. Periyasamy, K. Baskaran, A. Ilakkia, K. Vanitha, S. Selvaraj and D. Sakthisekaran, Cancer Chemother. Pharmacol., 2015, 75, 263-272.

48 K. Myer, Standard Handbook of Biomedical Engineering \& Design, McGRAW-HILL, New York, Chicago, San Francisco, Lisbon, London, Madrid, Mexico City, Milan, New Delhi, San Juan, Seoul, Singapore, Sydney, Toronto, 2003.

49 M. K. Yu, Y. Y. Jeong, J. Park, S. Park, J. W. Kim, J. J. Min, K. Kim and S. Jon, Angew. Chem., Int. Ed., 2008, 47, 53625365 .
50 K. Pombo García, K. Zarschler, L. Barbaro, J. A. Barreto, W. O'Malley, L. Spiccia, H. Stephan and B. Graham, Small, 2014, 10, 2516-2529.

51 G. Nardone, D. Compare, E. Liguori, V. Di Mauro, A. Rocco, M. Barone, A. Napoli, D. Lapi, M. R. Iovene and A. Colantuoni, Am. J. Physiol.: Gastrointest. Liver Physiol., 2010, 299, G669-G676.

52 A. Meerasa, J. G. Huang and F. X. Gu, Curr. Drug Delivery, 2011, 8, 290-298.

53 V. Mirabello, D. G. Calatayud, R. L. Arrowsmith, H. Ge and S. I. Pascu, Journal of Materials Chemistry B, 2015, 3, 56575672 . 